\title{
Dual TLR2 and TLR7 agonists as HIV latency-reversing agents
}

\author{
Amanda B. Macedo, ${ }^{1}$ Camille L. Novis, ${ }^{2}$ Caroline M. De Assis, ${ }^{1}$ Eric S. Sorensen, ${ }^{1}$ \\ Paula Moszczynski, ${ }^{1}$ Szu-han Huang, ${ }^{3}$ Yanqin Ren, ${ }^{3}$ Adam M. Spivak, ${ }^{4}$ R. Brad Jones, ${ }^{3}$ \\ Vicente Planelles, ${ }^{2}$ and Alberto Bosque ${ }^{1}$
}

'Department of Microbiology, Immunology, and Tropical Medicine, George Washington University, Washington, DC, USA. 2Division of Microbiology and Immunology, and Department of Pathology, Department of Medicine, University of Utah School of Medicine, Salt Lake City, Utah, USA. IInfectious Disease Division, Weill Cornell Medical College, New York, New York, USA. " Division of Infectious Diseases, Department of Medicine, University of Utah School of Medicine, Salt Lake City, Utah, USA.

The presence of a reservoir of latently infected cells in HIV-infected patients is a major barrier towards finding a cure. One active cure strategy is to find latency-reversing agents that induce viral reactivation, thus leading to immune cell recognition and elimination of latently infected cells, known as the shock-and-kill strategy. Therefore, the identification of molecules that reactivate latent HIV and increase immune activation has the potential to further these strategies into the clinic. Here, we characterized synthetic molecules composed of a TLR2 and a TLR7 agonist (dual TLR2/7 agonists) as latency-reversing agents and compared their activity with that of the TLR2 agonist Pam2CSK4 and the TLR7 agonist CS-9620. We found that these dual TLR2/7 agonists reactivate latency by 2 complementary mechanisms. The TLR2 component reactivates HIV by inducing NF-KB activation in memory $\mathrm{CD4}^{+} \mathrm{T}$ cells, while the TLR7 component induces the secretion of TNF- $\alpha$ by monocytes and plasmacytoid dendritic cells, promoting viral reactivation in $\mathrm{CD}^{+} \mathrm{T}$ cells. Furthermore, the TLR2 component induces the secretion of IL-22, which promotes an antiviral state and blocks HIV infection in CD4+ $T$ cells. Our study provides insight into the use of these agonists as a multipronged approach targeting eradication of latent HIV.

Conflict of interest: The authors have declared that no conflict of interest exists.

License: This work is licensed under the Creative Commons Attribution 4.0 International License. To view a copy of this license, visit http:// creativecommons.org/licenses/by/4.0/

Submitted: June 8, 2018 Accepted: August 23, 2018 Published: October 4, 2018

\section{Reference information:} JCI Insight. 2018;3(19):e122673. https://doi.org/10.1172/jci. insight.122673.

\section{Introduction}

Antiretroviral therapy (ART) effectively suppresses HIV replication and converts HIV from a lethal infection to a chronic and manageable disease (1-3). The major challenge that impedes HIV eradication in ARTtreated individuals is the establishment of a long-lived, replication-competent, and stable latent reservoir (4-10). Long-lived memory $\mathrm{CD} 4^{+} \mathrm{T}$ cells are thought to be the predominant cell type harboring latent virus (11-13). This latent reservoir is able to resume disease progression once ART is interrupted $(1,11,14,15)$. Because there is little or no expression of viral proteins, latently infected cells escape immune recognition and subsequent clearance. Current strategies aim to reverse HIV proviral latency using a latency-reversing agent (LRA), thus allowing the cytotoxic T cell arm of the immune system, or other immunotherapies, to clear residual infected cells. These strategies are collectively referred to as shock-and-kill (16). In this context, molecules that reactivate HIV and increase immune cell function of effector cells such as CD8 T cells and NK cells could help with the clearance of latently infected cells (17).

Toll-like receptors (TLRs) are pathogen-recognition receptors capable of sensing small molecular motifs conserved within microbes $(18,19)$. TLRs are present in various cellular compartments, including the plasma membrane, endosomes, lysosomes, and endolysosomes (20). These receptors have been detected on cells of both the innate and adaptive immune system (such as DCs, macrophages, granulocytes, T cells, B cells, NK cells, and mast cells) as well as endothelial and epithelial cells. Their engagement triggers signaling pathways that lead to production of inflammatory cytokines or pathways responsible for the induction of type I IFNs $(21,22)$.

Initial research using cell lines showed that TLR2, TLR5, TLR8, or TLR9 agonists are able to transactivate the HIV-1 long terminal repeat upon stimulation (23-26). Later, our group demonstrated that the TLR2 agonist Pam3CSK4 can reactivate latent HIV-1 in primary cells using an in vitro latency model 
and cells isolated from aviremic patients (27). Others have shown that TLR7 and TLR9 agonists are able to reactivate HIV by a mechanism involving immune activation of plasmacytoid DCs (pDCs) and NK cells and the secretion of soluble factors in an IFN- $\alpha$-mediated fashion $(28,29)$. Recently, a small clinical trial using the TLR9 agonist MGN1703 in patients under ART showed an increase in viral transcription, with minimal side effects (30). GS-9620 (vesatolimod) is an oral TLR7 agonist that has been in clinical trials for hepatitis B (31). This compound is currently under investigation in 2 clinical trials in HIV patients to target the latent reservoir (NCT02858401, NCT03060447). Moreover, in vivo injections of GS-9620 and the analog GS-986 induced viremia and led to reductions in viral reservoirs in SIV-infected macaques under ART (32). A recent report at the 2018 Conference on Retroviruses and Opportunistic Infections (CROI) showed that the combination of GS-9620 with the broadly neutralizing antibody PGT-121 promoted long-term remission in an SIV-infected rhesus monkey macaque model under ART (Abstract number 73LB; http://www.croiconference.org/sites/ default/files/uploads/73LB.pdf). However, the mechanism by which TLR7 engagement leads to the viral reactivation of latently infected $\mathrm{CD} 4^{+} \mathrm{T}$ cells has not been elucidated.

The present study was designed to test whether targeting TLR2 and TLR7 simultaneously would have combined and favorable effects on the latent reservoir. For this purpose, we evaluated several commercially available, synthetic, dual TLR agonists that were formulated as combinations of a TLR2 and a TLR7 agonist. These compounds were designed to enhance immune responses through the activation of TLRs with different downstream pathways. Our results indicate that dual TLR2/7 agonists have multiple advantages over single TLR2 or TLR7 agonists. First, they can directly reactivate latent HIV in primary $\mathrm{CD}^{+} \mathrm{T}$ cells by inducing NF- $\mathrm{KB}$ activation in a TLR2-dependent manner. Second, like TLR2 agonists, dual agonists also mediate the secretion of IL-22. IL-22 is then able to inhibit de novo HIV infection of $\mathrm{CD}^{+} \mathrm{T}$ cells. Third, we demonstrated that dual agonists preserve the ability of TLR7 agonists, including GS-9620, to reactivate latent HIV, which is mediated in part by the secretion of TNF- $\alpha$ by monocytes and pDCs. Overall, this work further elucidates the mechanism by which TLR7 agonists reactivate latent HIV and identifies dual TLR2/7 agonists as LRA candidates for shock-and-kill strategies towards HIV cure.

\section{Results}

Ability of dual TLR2/ 7 agonists to reactivate latent HIV in vitro. Our group and others have previously shown that TLR2 agonists can directly reactivate latent $\mathrm{HIV}$ in primary $\mathrm{CD} 4^{+} \mathrm{T}$ cells $(23,27,33,34)$. Based on these findings, we wanted to address whether synthetic molecules formulated to combine a TLR2 agonist and the TLR7 agonist 8-hydroxyadenine could also reactivate latent HIV. We selected 4 commercially available synthetic agonists with different structures (Supplemental Figure 1; supplemental material available online with this article; https://doi.org/10.1172/jci.insight.122673DS1). These dual TLR agonists were formulated to preserve both their TLR2 and TLR7 activity, as demonstrated in cell lines stably expressing an NF- $\mathrm{BB}$-inducible secreted embryonic alkaline phosphatase (SEAP) reporter gene and human TLR2 or TLR7 (https://www.invivogen.com/c1531\#specifications). CL401 is a molecule composed of Pam2C conjugated with 8-hydroxyadenine. Pam2C lacks the serine and 4-lysine polypeptide of the TLR2 agonist Pam2CSK4. Pam2CSK4 is a synthetic diacylated lipopeptide that signals through TLR2/6 heterodimers or TLR2 homodimers (35). CL413 and CL531 contain the full Pam2CSK4 conjugated to 8-hydroxyadenine either via the terminal acid function of Pam2CSK4 (CL413) or the lateral chain of the second lysine of Pam2CSK4 (CL531) (Supplemental Figure 1). Finally, we also selected CL572, which combines 8-hydroxyadenine with monoacyl-ethyl-cysteine via a glutamic acid derivative. Monoacyl-ethyl-cysteine-containing dipeptides have been shown to specifically activate human but not mouse TLR2 (36).

Initially, we tested the ability of these synthetic molecules to reactive latent HIV in a TLR2-dependent manner. For that purpose we used a derivative of the cell line JLAT10.6 that had been stably transduced with a previously described TLR2 expression construct (JLAT-TLR2) $(33,37)$. We observed that each of the synthetic dual TLR2/ 7 agonists tested were able to reactivate latent HIV in JLAT-TLR2 cells, but not in the control JLAT10.6 cell line at $1 \mu \mathrm{M}$ (Figure 1A). The TLR7 agonists CL264 and GS-9620 were unable to reactivate latent HIV in this cell model of latency (Figure 1A). We next performed a dose-response study to compare the relative potency of these TLR agonists. Pam2CSK4 was the most potent agonist, with an EC50 of $5 \mathrm{pM}$ (Figure 1B). The addition of 8-hydroxyadenine to Pam2CSK4 reduced the potency of CL531 and CL413 to the low nanomolar range (EC50 of 3 and $48.1 \mathrm{nM}$, respectively). CL572, containing the monoacyl-ethyl-cysteine derivative, had an EC50 of 385 nM (Figure 1B). CL401 and Pam3CSK4 had the lowest potency of all the analogs tested (Figure 1B). In conclusion, all the dual TLR2/7 agonists tested 

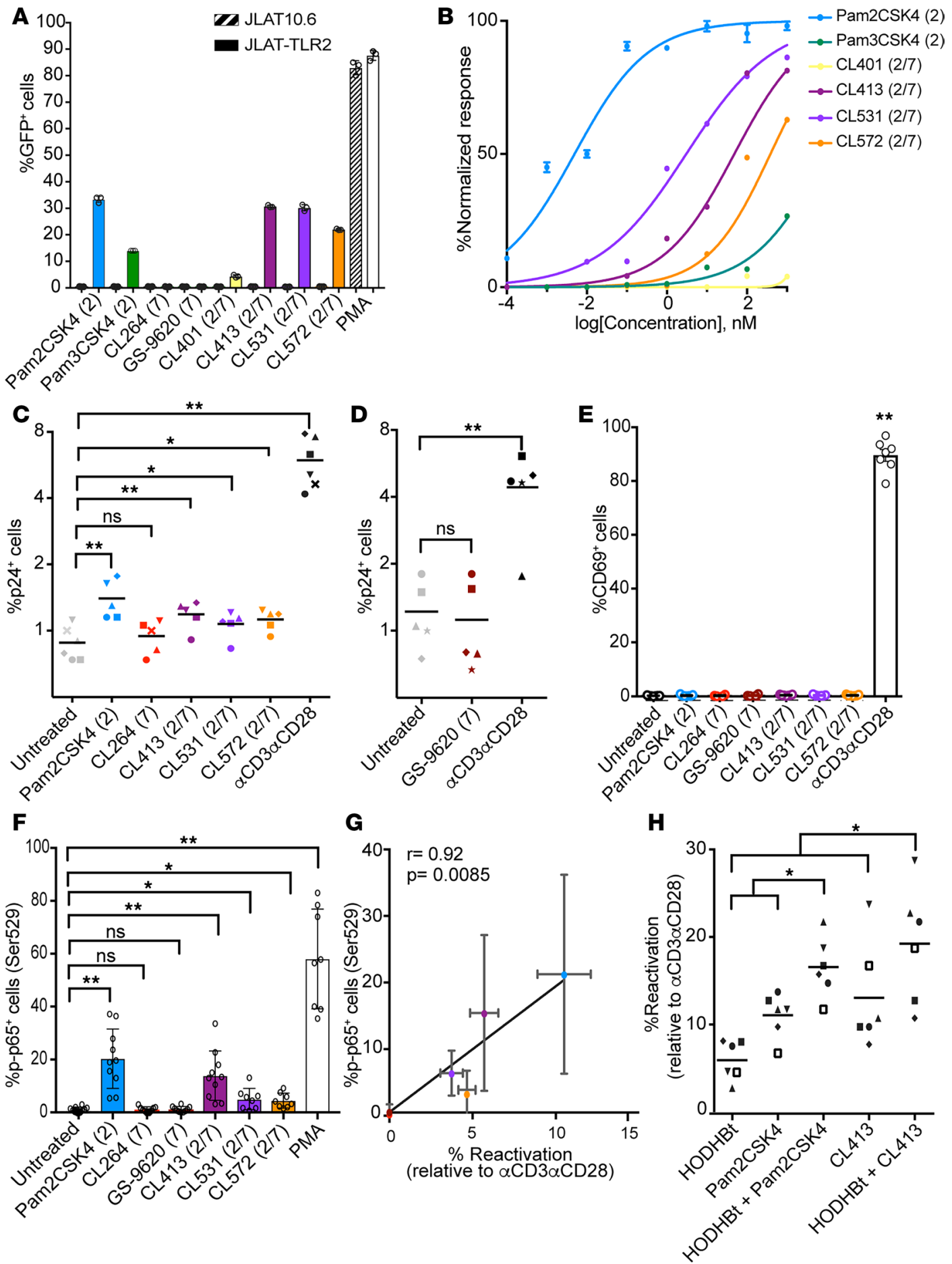

Figure 1. Dual TLR2/7 agonists induce HIV reactivation in latently infected CD4+ $\mathbf{T}$ cells. (A) Viral reactivation mediated by TLR agonists in JLAT10.6 and JLAT-TLR2. Data represent the mean \pm SD of a representative experiment from 3 independent experiments performed in triplicate. (B) Doseresponse of Pam2CSK4, Pam3CSK4, CL401, CL413, CL531, and CL572 ranging from $0.1 \mathrm{pM}$ to $1 \mu \mathrm{M}$ in JLAT-TLR2. Data represent the mean \pm SD of a representative experiment from 3 independent experiments preformed in triplicate. (C and D) Reactivation of latent HIV in the Tcm model with IL-2 alone (untreated), IL-2 plus $1 \mu \mathrm{M}$ of the indicated TLR agonist or $\alpha$ CD3 $\alpha$ CD28 $(n=5)$. (E) Expression of CD69 in total isolated CD4+ T cells treated with the indicated TLR agonist or $\alpha \operatorname{CD} 3 \alpha \operatorname{CD} 28(n=3)$. Data represent the mean \pm SD. (F) Percentage of p65 phosphorylation on serine 529 in memory CD4 ${ }^{+}$ T cells after 15 minutes of stimulation with the indicated TLR agonist or PMA $(n=8-10)$. Data represent the mean \pm SD. (C) Spearman's correlation of the levels of phosphorylated p65 with the normalized reactivation levels in the Tcm model. Data represent the mean \pm SD. (H) Reactivation of latent HIV in the Tcm model induced by HODHBt at $100 \mu \mathrm{M}$ alone or combined with $1 \mu \mathrm{M}$ Pam2CSK4 or $1 \mu \mathrm{M}$ CL413; values were normalized relative to $\alpha \operatorname{CD} 3 \alpha \operatorname{CD} 28(n=6) .{ }^{*} P<0.05,{ }^{* *} P<0.01$ by 2-tailed Wilcoxon's matched-pairs signed-rank test for all comparisons. ns, not significant. 
retain the ability to reactivate latent HIV in a TLR2-mediated manner in the low nanomolar range. Since the dual TLR CL401 presented low activity as a TLR2 agonist, we excluded it from subsequent studies and selected CL413, CL531, and CL572 for further studies.

Next, we tested the ability of CL413, CL531, and CL572 to reactivate latent HIV in the cultured central memory $\mathrm{T}$ cell $(\mathrm{Tcm})$ model of latency $(38,39)$. Briefly, this primary cell model is based on the generation of latently infected $\mathrm{CD}^{+}$Tcm cells by infection with the replication-competent molecular clone $\mathrm{HIV}_{\text {NL4-3 }}$ following antiretroviral suppression (38). In this primary cell model, latency reversal is measured by the induction of p24 Gag protein and by the surface downregulation of CD4 expression by the accessory genes Nef and $\mathrm{Vpu}(40,41)$. Altogether, this combination of readouts ensures the ability of the LRAs to induce productive transcription because they evaluate the presence and function of several viral proteins (Nef, Vpu, and Gag) (42). Pam2CSK4, CL413, CL531, and CL572 induced reactivation of latent HIV when compared with untreated control $(P<0.05$, Figure 1C). In contrast, neither of the TLR7 agonists tested, CL264 or GS-9620, induced viral reactivation in this primary cell model (Figure 1, C and D). We next evaluated whether these agonists induced $\mathrm{T}$ cell activation. To do that, we measured the induction of the early activation marker CD69 on total isolated CD4 ${ }^{+} \mathrm{T}$ cells. While anti-CD3/anti-CD28 $(\alpha \mathrm{CD} 3 \alpha \mathrm{CD} 28)$ strongly induced CD69 expression, none of the agonists induced the expression of this activation marker (Figure 1E). In summary, dual TLR2/7 agonists reactivate latently infected CD4 ${ }^{+} \mathrm{T}$ cells without apparent induction of $\mathrm{CD}^{+} \mathrm{T}$ cell activation.

We have previously described that TLR2 agonists reactivate latent HIV via activation of NF-кB (27). We hypothesized that the ability of these agonists to reactivate latent $\mathrm{HIV}$ in $\mathrm{CD} 4^{+} \mathrm{T}$ cells was due to their differential ability to activate NF- $\mathrm{kB}$. To test this hypothesis, we measured levels of phosphorylation at serine 529 (Ser529) in the NF-кB subunit p65 (p-p65) by phosphoflow in isolated memory CD4 ${ }^{+} \mathrm{T}$ cells. Phosphorylation of Ser529 in $\mathrm{p} 65$ has been shown to increase the transcriptional activity of NF- $\mathrm{B}$ $(43,44)$. The different TLR2 agonists were able to induce p65 phosphorylation in memory CD4 ${ }^{+} \mathrm{T}$ cells compared with untreated control but to a lesser degree than the positive control PMA (Figure 1F). None of the TLR7 agonists tested were able to induce p65 phosphorylation in primary memory $\mathrm{CD}^{+} \mathrm{T}$ cells (Figure 1F). Furthermore, the induction of p65 phosphorylation levels in memory CD4 ${ }^{+} \mathrm{T}$ cells strongly correlated with the ability of the different agonists to reactivate latent HIV in the primary Tcm model (Figure 1G). These data further confirm that in contrast with TLR2 agonists, TLR7 agonists do not exert their latency reactivation properties by directly targeting $\mathrm{CD} 4^{+} \mathrm{T}$ cells.

We have shown that TLR2 agonists can reactivate latent HIV in a primary cell model of latency, albeit to a modest degree. This is a common feature of most LRAs used in the clinical setting (45). It has been proposed that combinations of LRAs with different mechanisms of action may be needed to efficiently reactivate latent HIV (45). For this reason, we performed an unbiased screen of 94 epigenetic modulators and assessed their ability to synergize with a TLR2 agonist. For that purpose, we used JLAT-TLR2 as a screening tool. Compounds were tested at $10 \mu \mathrm{M}$, either alone or in the presence of the TLR-2 agonist CL572 $(1 \mu \mathrm{M})$. Cell viability and viral reactivation were assessed by flow cytometry (Supplemental Figure 2, A and B). Synergies between the epigenetic modulators and the TLR2 agonist were calculated using Bliss independence model analysis (46) (Supplemental Figure 2C). From this screening, we selected 6 compounds that presented low toxicity and high levels of synergy with the TLR2 agonist and have been previously shown to induce HIV reactivation. We selected the histone deacetylase inhibitors entinostat (MS275) and chidamide (47, 48); the bromodomain inhibitors JQ-1, PFI-1, and I-CBP112 (49, 50); and the DNA methyltransferase inhibitor decitabine (51). We first confirmed that these 6 epigenetic modulators can increase viral reactivation mediated by the TLR2 agonist Pam2CSK4 in the JLAT-TLR2 model (Supplemental Figure 3A). Next, we evaluated the ability of these 6 epigenetic modulators to reactivate latent HIV in the cultured Tcm model of latency, either alone or in combination with Pam2CSK4. Although these compounds had some latency-reversing activity in this model (around $10 \%$ of that of maximal stimulation), none of them enhanced the viral reactivation mediated by Pam2CSK 4 in this primary cell model (Supplemental Figure 3B). We next tested whether Pam2CSK4 and the most potent dual TLR agonist, CL413, could be used in combination with 3-Hydroxy-1,2,3-benzotriazin-4(3H)-one (HODHBt), a benzotrazin derivative previously shown to reactivate latent HIV in a STAT5-dependent manner (52). The combination of HODHBt plus Pam2CSK4 or CL413 increased viral reactivation compared with each stimulation alone (Figure 1H). These results suggest that TLR agonists may have the potential to be used in combination with other LRAs to increase the efficacy of these strategies towards reactivating latent HIV. 
A

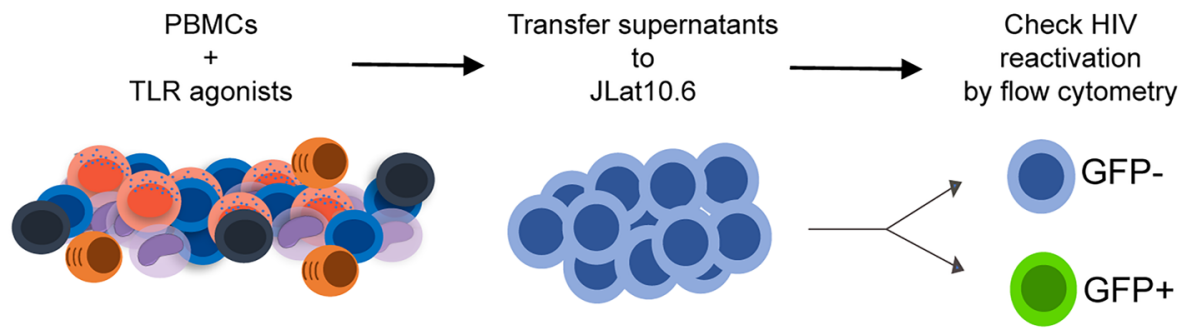

B

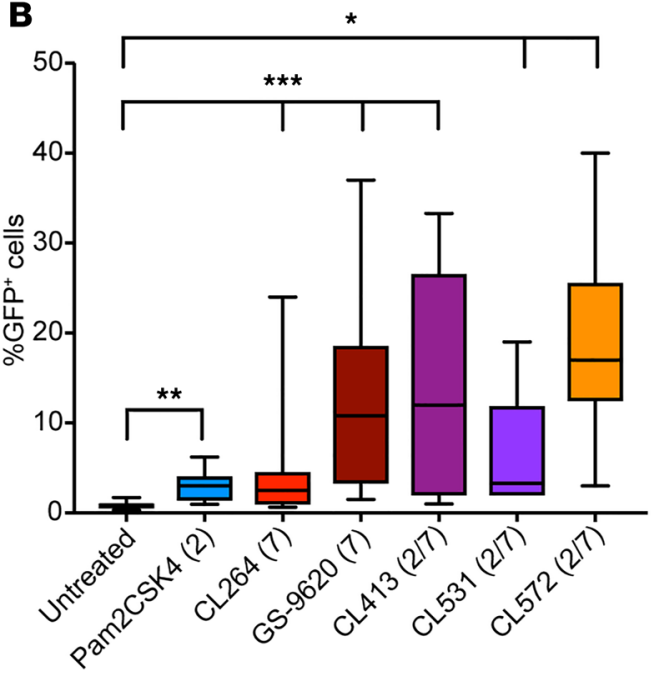

C

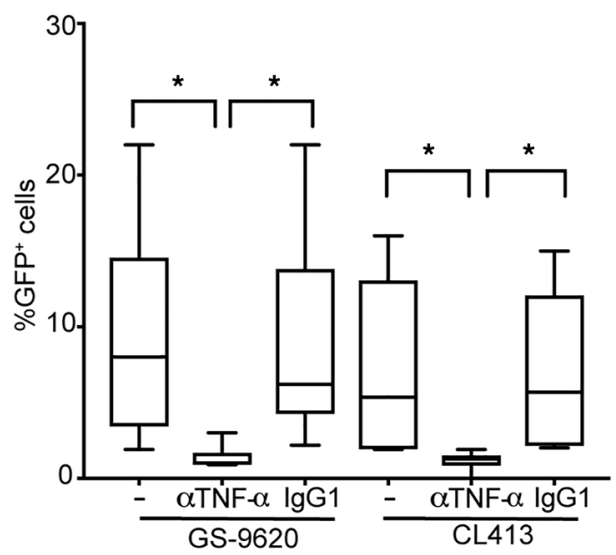

D

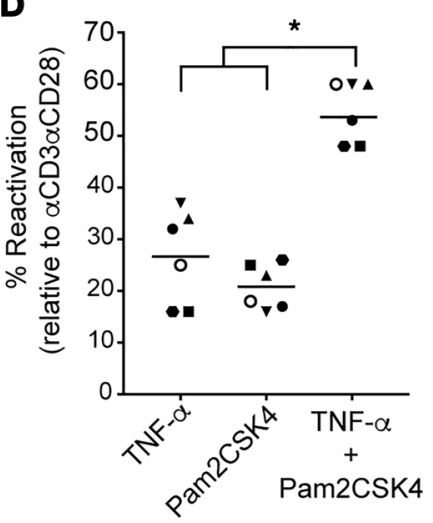

E

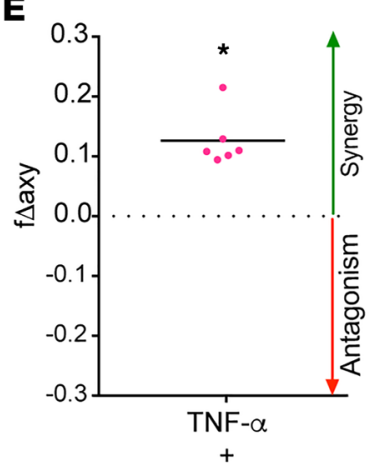

Pam2CSK4
F

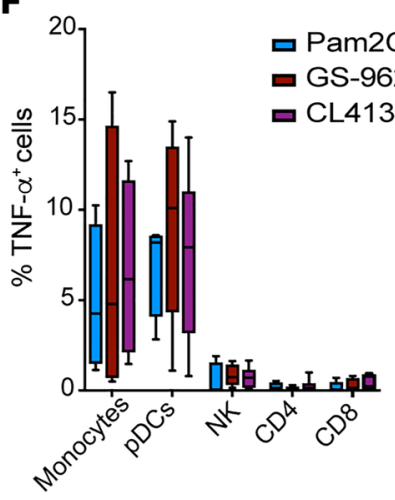

G
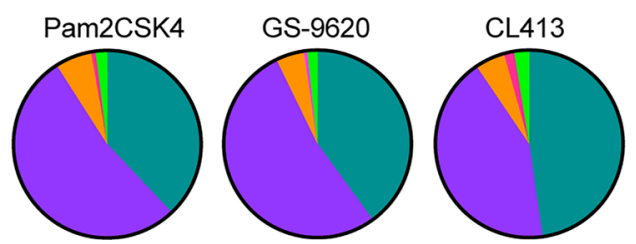

$\square$ Monocyte
$\square$ pDC
$\square$ NK
$\square$ CD4
$\square$ CD8

Figure 2. The ability TLR7 agonists to reactivate HIV is mediated by TNF- $\alpha$. (A) Schematic of the assay. (B) Box-and-whisker plots indicating the percentage viral reactivation in JLAT10.6 cells incubated with supernatants collected from PBMCs stimulated with the TLR agonists indicated $(n=9-12)$. Error bars indicate maximum and minimum. (C) Box-and-whisker plots indicating viral reactivation mediated by supernatants from PBMCs treated with CS-9620 and CL413 preincubated with a monoclonal antibody against TNF- $\alpha$ or an isotype control $(n=6)$. Error bars indicate maximum and minimum. (D) Viral reactivation of latent HIV in the Tcm model with TNF- $\alpha$, Pam2CSK4, or a combination of the two $(n=6)$. Data were normalized relative to the reactivation mediated by $\alpha C D 3 \alpha C D 28$. (E) Calculation of synergy for LRA combination using the Bliss independence model. Data are presented as the difference between the observed and predicted fractional response. Statistical significance for the experimental $f$ was calculated using a ratio paired $t$ test compared with the predicted $f_{\mathrm{a}}$ for the combination $(n=6)$. (F) PBMCs treated with Pam2CSK4, GS-9620, and CL413 overnight were stained for cell-type specific markers and TNF- $\alpha(n=6)$. Data represent the mean \pm SD. (G) Pie chart visualization of the relative contribution of each cell type to the total TNF- $\alpha$ response for each stimulation $(n=6) .{ }^{*} P<0.05,{ }^{* *} P<0.001$ by 2 -tailed Wilcoxon's matched-pairs signed-rank test. 
TLR7 agonists reactivate latent HIV in a TNF- $\alpha$-dependent manner. It has been described that the TLR7 agonist GS-9620 and the TLR9 agonist MGN-1703 reactivate latent HIV in CD4 ${ }^{+} \mathrm{T}$ cells in a paracrine manner through the activation of immune cells and the secretion of soluble factors, or in a direct manner in monocytic cell lines $(26,28,29)$. We therefore asked whether dual TLR agonists maintain the ability to reactivate latent HIV in a TLR7-dependent manner. To answer this question, we developed an in vitro assay to test if soluble factors released by peripheral blood mononuclear cells (PBMCs) cultured in the presence of different TLR agonists have the potential to reactivate latent HIV (Figure 2A). We decided to use JLAT10.6 as target cells, as this cell line is unresponsive to stimulation with the TLR agonists used in this study (Figure 1A). By this method, the viral reactivation observed is mediated exclusively through the action of soluble products secreted in the supernatants by the treated PBMCs. As shown in Figure 2B, all the supernatants from stimulated PBMCs tested induced significant levels of HIV-reactivation in JLAT10.6 cells compared with supernatants from untreated cells, albeit to different degrees. Supernatants from PBMCs treated with either Pam2CSK4 or the TLR7 agonist CL264 induced the lowest levels of viral reactivation. In spite of the inability of GS-9620 to directly reactivate latent HIV in CD4 ${ }^{+} \mathrm{T}$ cells, supernatants of PBMCs treated with this agonist effectively reactivate latent HIV. Finally, we observed that the supernatants from PBMCs treated with any of the dual TLR agonists can induce HIV reactivation. CL413, CL531, and CL572 induced statistically significant higher levels of reactivation than the individual agonist Pam2CSK4. In addition, CL413 and CL572 generated significantly higher viral reactivation than CL264 (Figure 2B). We next wanted to evaluate whether the synthetic coupling of the TLR2 and TLR7 agonists had an advantage over each single agonist administered together. For that purpose, supernatants from untreated PBMCs or those treated with Pam2CSK4, CL264, a combination of both, or CL413 were tested for their ability to reactivate JLAT10.6. Our results suggest that the synthetic coupling of these agents has an advantage over each stimulation alone (Supplemental Figure 4). Overall, these results indicate that the dual TLR agonists maintain the ability to reactivate latent HIV in a TLR7-dependent manner to a similar degree as GS-9620.

We then set out to identify the soluble factor(s) responsible for the viral reactivation observed in JLAT10.6 upon transfer of supernatants. First, we tested whether different cytokines reported to be induced by TLR agonists were able to induce viral reactivation in JLAT10.6 (28, 29, 53). We tested IL-6, IL-10, IFN- $\gamma$, IFN- $\alpha$, IFN- $\omega$, TNF- $\alpha$, IP-10, and IL-22. Among all 8 cytokines tested, TNF- $\alpha$ was the only cytokine able to induce viral reactivation in JLAT10.6 (Supplemental Figure 5). This result was not unexpected as this cell line was clonally selected based on their ability to reactivate latent HIV in a TNF- $\alpha-$ mediated manner (37). To confirm whether TNF- $\alpha$ was responsible for the HIV reactivation observed in this assay, we added a monoclonal antibody against TNF- $\alpha$ to the supernatants. Supernatants treated with the anti-TNF- $\alpha$ monoclonal antibody lost the ability to reactivate JLAT10.6, but not those treated with isotype control (Figure 2C). Next, we tested whether TNF- $\alpha$ alone or combined with Pam2CSK4 can reactivate latent HIV in the cultured Tcm model of latency. In this primary cell model, TNF- $\alpha$ induced viral reactivation to a similar extent as Pam2CSK4 (Figure 2D). A combination of TNF- $\alpha$ and Pam2CSK4 showed a higher viral reactivation than each of the agonists alone (Figure 2D). To evaluate whether the observed viral reactivation seen in this combination was synergistic, we performed Bliss independence model analysis (46). This analysis confirmed the synergistic ability of Pam2CSK4 and TNF- $\alpha$ to induce viral reactivation (Figure 2E). Altogether, these results confirm that TLR7 and the dual TLR agonists can induce the secretion of TNF- $\alpha$ from PBMCs and that TNF- $\alpha$ is the cytokine responsible for the paracrine viral reactivation by TLR7 agonists observed in our experimental system.

We were interested to investigate the cell subsets responsible for the TNF- $\alpha$ production upon TLR2 and TLR7 stimulation. We then measured the induction of TNF- $\alpha$ intracellularly by intracellular cytokine staining after challenging PBMCs with different TLR agonists. Among the different cell subsets analyzed, monocytes and pDCs were the main subsets producing TNF- $\alpha$ with the 3 agonists tested (Figure $2 \mathrm{~F}$ ). Both GS-9620 and CL413 induced higher levels of TNF- $\alpha$ than Pam2CSK4. This was in agreement with their higher ability to reactivate latent HIV (Figure 2B). In addition, we determined the contribution of distinct cell types to the total production of TNF- $\alpha$ mediated by each agonist (Figure $2 \mathrm{G}$ ). pDCs and monocytes were the cell subsets that accounted for the majority of the TNF- $\alpha$ production. NK cells contributed an average of $5 \%$ of the total response. Finally, $\mathrm{CD} 8^{+}$and $\mathrm{CD} 4^{+} \mathrm{T}$ cells accounted for the smallest portion of the total TNF- $\alpha$ production (Figure $2 \mathrm{G}$ ). We have shown that the dual TLR agonist CL413 induced higher levels of viral reactivation than each of the agonists alone (Supplemental Figure 4). We then wanted to address whether a similar pattern can be observed for the secretion of TNF- $\alpha$. The same supernatants 
used in Supplemental Figure 4 were tested for the presence of TNF- $\alpha$. CL413 induced higher levels of TNF- $\alpha$ than each of the agonists, either alone or combined (Supplemental Figure 6A). As expected, the ability of these supernatants to reactivate latent HIV in JLAT10.6 correlated with the concentration of TNF- $\alpha$ in the same supernatants (Supplemental Figure 6B). These results indicate that TNF- $\alpha$ is one of the factors secreted by monocytes and pDCs after treatment with TLR2, TLR7, and dual TLR2/7 agonists that promotes viral reactivation from latency. Furthermore, we demonstrated that the dual TLR2/7 agonist CL413 maintains similar properties compared with GS-9620 in the ability to induce secretion of TNF- $\alpha$ and reactivate HIV in a paracrine manner.

Biological sex is not an intrinsic biological variable in TLR-induced HIV reactivation. pDCs isolated from female donors have been shown to be more sensitive to TLR7 ligands than those from male and produce higher levels of IFN- $\alpha$ upon TLR7 stimulation $(54,55)$. These results may suggest that the use of TLR agonists as LRAs may be influenced by the biological sex of the participant. We therefore decided to compare the responses between male and female donor cells to the different TLR agonists. First, we analyzed whether biological sex influenced the reactivation of latent HIV mediated by Pam2CSK4 in the Tcm model of latency. In this primary cell model, we did not observe any difference in the ability of Pam2CSK4 to reactivate latent HIV between female and male donors (Supplemental 7A). In addition, we did not see a correlation between the ability of Pam2CSK4 to reactivate latently infected Tcm cells with the age of the donors (Supplemental 7B). Next, we analyzed whether the latent reactivation observed in JLAT10.6 mediated by the supernatants of TLR agonist-treated PBMCs is influenced by the biological sex and age of the donors. Supernatants of PBMCs treated with either GS-9620 or CL413 were equally able to reactivate latent HIV whether the donor cells were of female or male origin and independent of the age of the donors (Supplemental Figure 7, C-F). Together, these data suggest that biological age and sex are not intrinsic variables in the ability of these TLR agonists to reactivate latent HIV.

Dual TLR agonists maintain the ability to promote immune effector functions. GS-9620 has been shown to induce $\mathrm{CD}^{+} \mathrm{T}$ and $\mathrm{NK}$ cell activation that promotes HIV-specific immune responses (28). To further characterize whether the dual TLR2/7 agonists preserve these functions, we treated PBMCs from either HIV-suppressed participants or healthy donors with the different TLR agonists in the presence of ART for 4 days. At 48 hours, we assessed global toxicity as well as the induction of the activation marker CD69 in $\mathrm{NK}, \mathrm{CD}^{+}$, and $\mathrm{CD} 8^{+} \mathrm{T}$ cells. There was not a statistically significant difference when comparing cells from HIV-negative donors (black squares) and aviremic participants (gray circles) $(P>0.05$, Mann-Whitney test) (Figure 3, A-D). For simplicity, the results were combined and a unique mean is depicted in the graphs. First, we did not observe any global toxicity when PBMCs were treated with any of the TLR agonists tested (Figure 3A). In contrast, the positive control for T cell activation, PMA/ionomycin, drastically compromised the viability of the cultures (Figure 3A). All the TLR agonists tested induced significant upregulation of CD69 in NK cells compared with untreated control (Supplemental Table 1). In agreement with Tsai et al. (28), we observed a strong NK activation with GS-9620. And here, dual TLR2/7 agonists induced levels comparable to that of GS-9620 (Figure 3B). Despite our previous observation that these agonists did not induce $\mathrm{CD}^{+} \mathrm{T}$ cell activation when cells were treated in isolation (Figure 1E), we observed a low but statistically significant induction of CD69 in $\mathrm{CD}^{+} \mathrm{T}$ cells when PBMCs were stimulated with TLR agonists (Figure 3C and Supplemental Table 2). Similar results were obtained for $\mathrm{CD} 8^{+} \mathrm{T}$ cells (Figure 3D and Supplemental Table 3). In conclusion, dual TLR agonists preserve their ability to induce NK activation with minimal activation of $\mathrm{CD}^{+}$and $\mathrm{CD} 8^{+} \mathrm{T}$ cells and to a similar extent as GS-9620. This NK activation was previously reported to be beneficial for HIV clearance (29).

Dual TLR agonists induce the secretion of proinflammatory and antiviral cytokines. In order to fully characterize the cytokine profile induced by these agonists, we measured a panel of 14 cytokines released by PBMCs from HIV-negative donors and aviremic participants in the presence of the different TLR agonists using a cytometric bead-based multiplex system and ELISA (see Methods). As in Figure 3, the results were combined and a unique mean is depicted in the graphs (Figure 4). Among the 14 cytokines measured, IL-6, IL-10, IFN- $\gamma$, IL-22, and IFN- $\alpha$ were the only cytokines detected above the limit of detection. All the TLR agonists tested induced the secretion of IL-6 (Figure 4A). Dual TLR agonists induced similar levels of IL-6 compared with the TLR2 agonist Pam2CSK4 but significantly higher levels when compared with the TLR7 agonists CL264 and GS-9620 (Supplemental Table 4). Dual TLR agonists induced similar levels of IL-10 compared with the TLR7 agonists CL264 and GS-9620 but higher than Pam2CSK4 (Figure 4B and Supplemental Table 5). IFN- $\gamma$ was induced by all the agonists except CL264 and showed statistically significant increased 
A

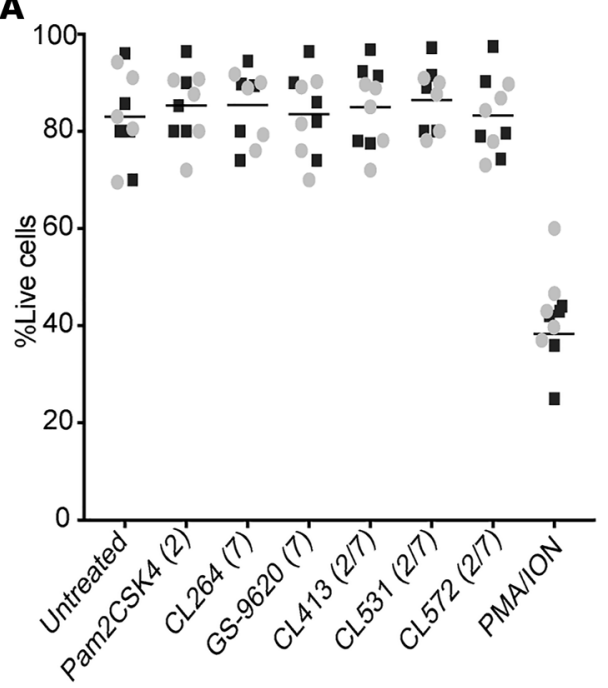

C

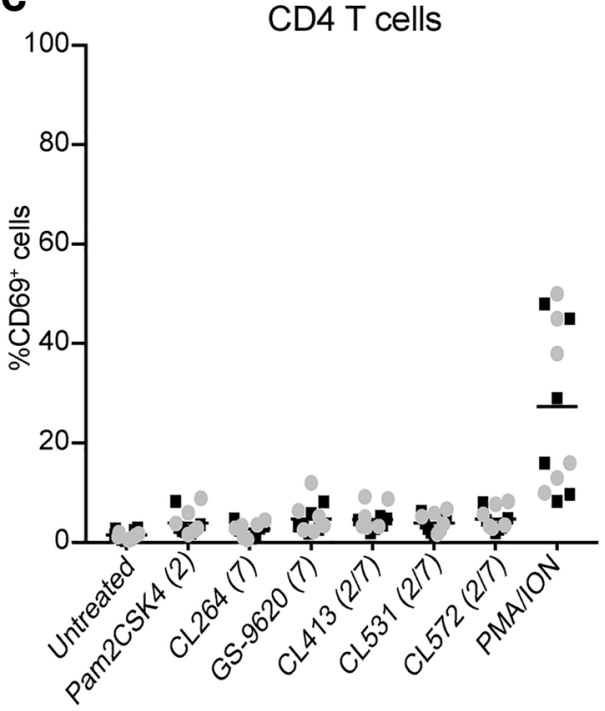

B

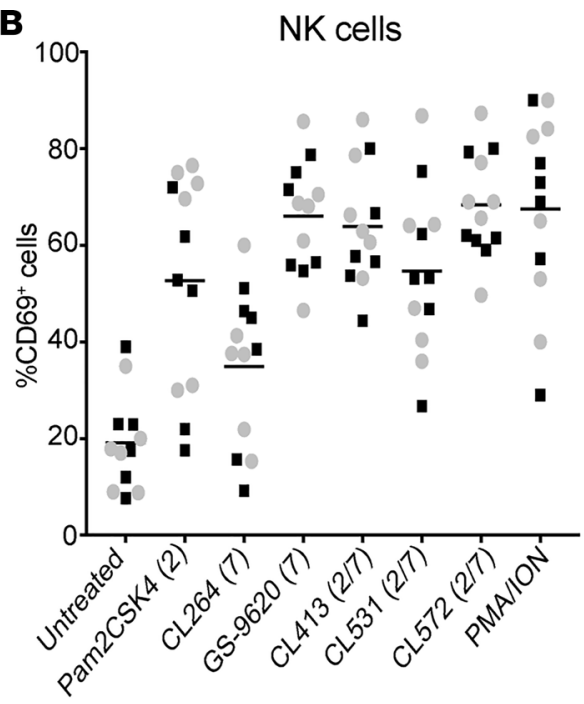

D

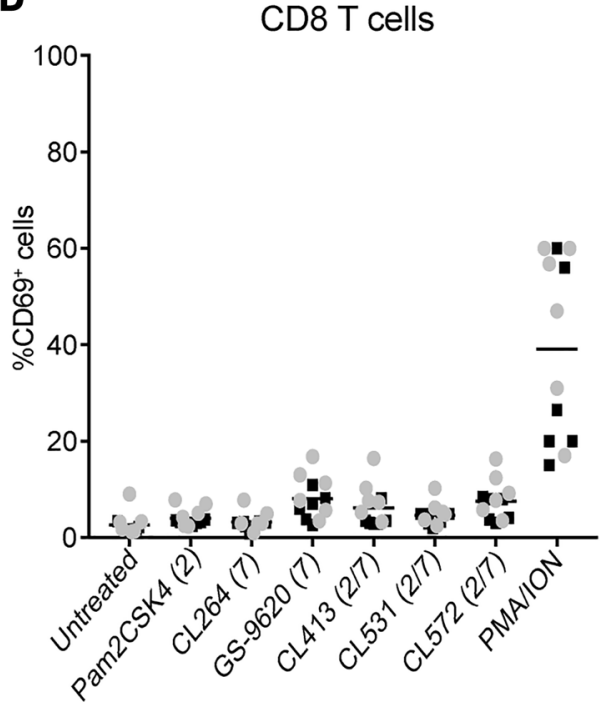

Figure 3. Dual TLR agonists induce NK cell activation without overt toxicity. (A) Percentage of live cells after 48-hour incubation of PBMCs with $1 \mu \mathrm{M}$ of the indicated TLR agonists or PMA plus ionomycin (PMA/ION). Gray circles represent aviremic participants and black squares HIV-negative donors. For simplicity the results were combined and a unique mean is depicted ( $n=6$ each group). (B-D) CD69 induction of (B) NK cells, (C) CD4+ T cells, and (D) CD8 ${ }^{+}$ T cells ( $n=6$ each group). Statistical analysis is provided in Supplemental Tables. Mann-Whitney $U$ test was used for comparisons between HIV-negative donors and aviremic participants and 2-tailed Wilcoxon's matched-pairs signedrank test was used to compare stimuli.

levels compared with untreated control (Figure 4C and Supplemental Table 6). Pam2CSK4 and all 3 dual TLR2/7 agonists induced significantly higher levels of IL-22 compared with untreated control (Figure $4 \mathrm{D}$ and Supplemental Table 7). Interestingly, IL-22 was the only measured cytokine that dual TLR2/7 agonists induced to statistically significant higher levels compared with Pam2CSK4 or GS-9620 (Figure 4D and Supplemental Table 7). Finally, we could observe statistically significant higher levels of IFN- $\alpha$ over untreated condition after stimulation with all the agonists and dual TLR2/7 agonists induced this cytokine to levels comparable to GS-9620 (Figure 4E and Supplemental Table 8).

It has been previously shown that GS-9620 is able to induce extracellular HIV RNA and expression of SIV RNA ex vivo $(28,32)$. We have shown that the dual TLR2/7 agonists evaluated in this study can induce viral protein expression in the primary cell model of latency (Figure 1C). To address whether the dual TLR2/7 agonists and GS-9620 were also able to induce HIV protein expression in cells isolated from aviremic participants, we collected the supernatants from 8 HIV-suppressed participants and measured 

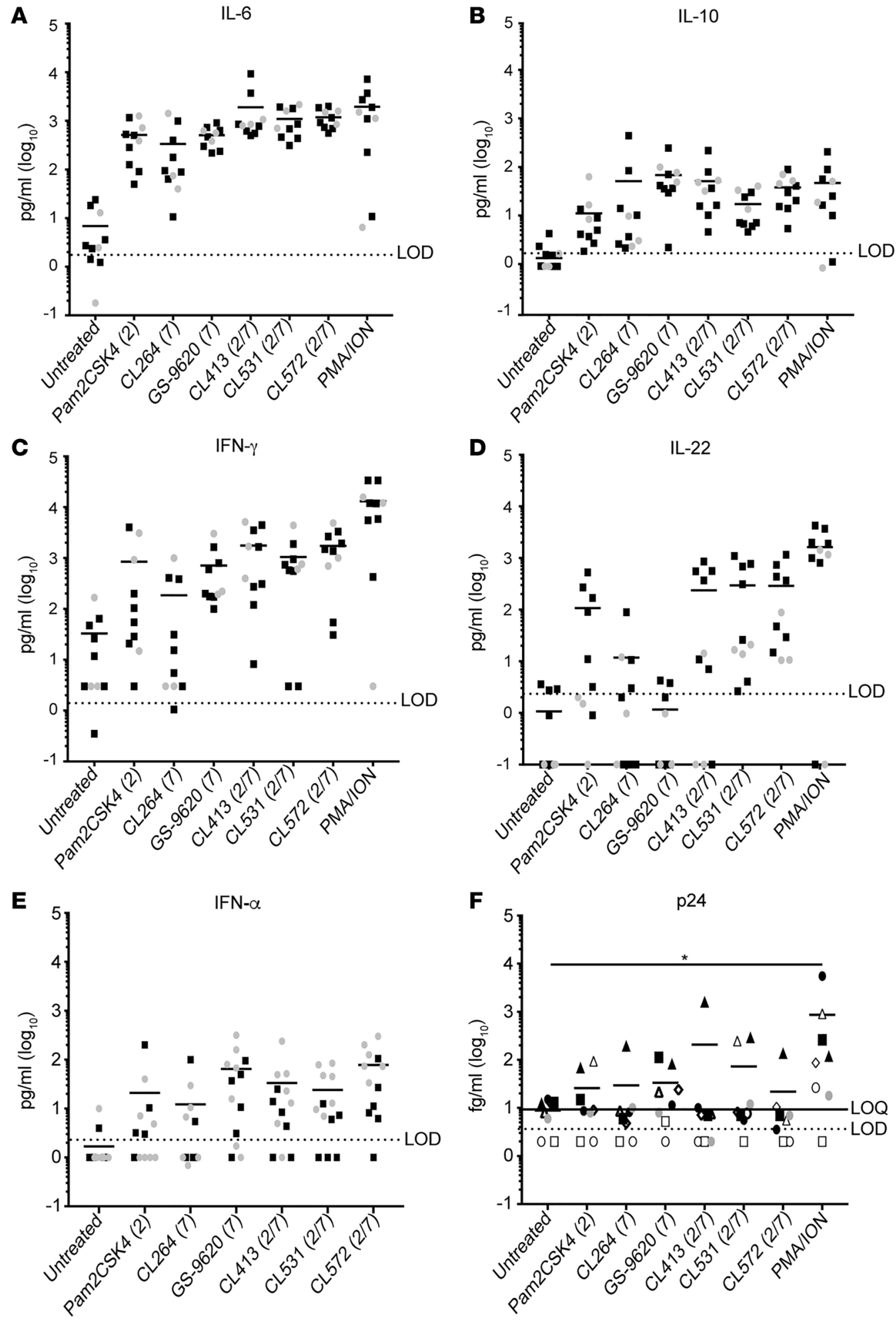

Figure 4. Dual TLR agonists induce secretion of proinflammatory and antiviral cytokines. Levels of IL- 6 (A), IL-10 (B), IFN- $\gamma$ (C), IL-22 (D), and IFN- $\alpha$ (E) were quantified in cell culture supernatants after treatment of PBMCs isolated from aviremic participants and HIV-negative donors with $1 \mu M$ of indicated TLR agonists or PMA plus ionomycin (PMA/ION) for 3 days. (F) p24 Gag protein was quantified in cell culture supernatants from PBMCs isolated from ART-suppressed HIV-infected individuals and treated with TLR agonists for 4 days using a digital ELISA $(n=8)$. Statistical analysis is provided in Supplemental Tables. Mann-Whitney $U$ test was used for comparisons between HIV-negative donors and aviremic participants and 2-tailed Wilcoxon's matched-pairs signed-rank test was used to compare stimuli. ${ }^{*} P<0.05$. 
A
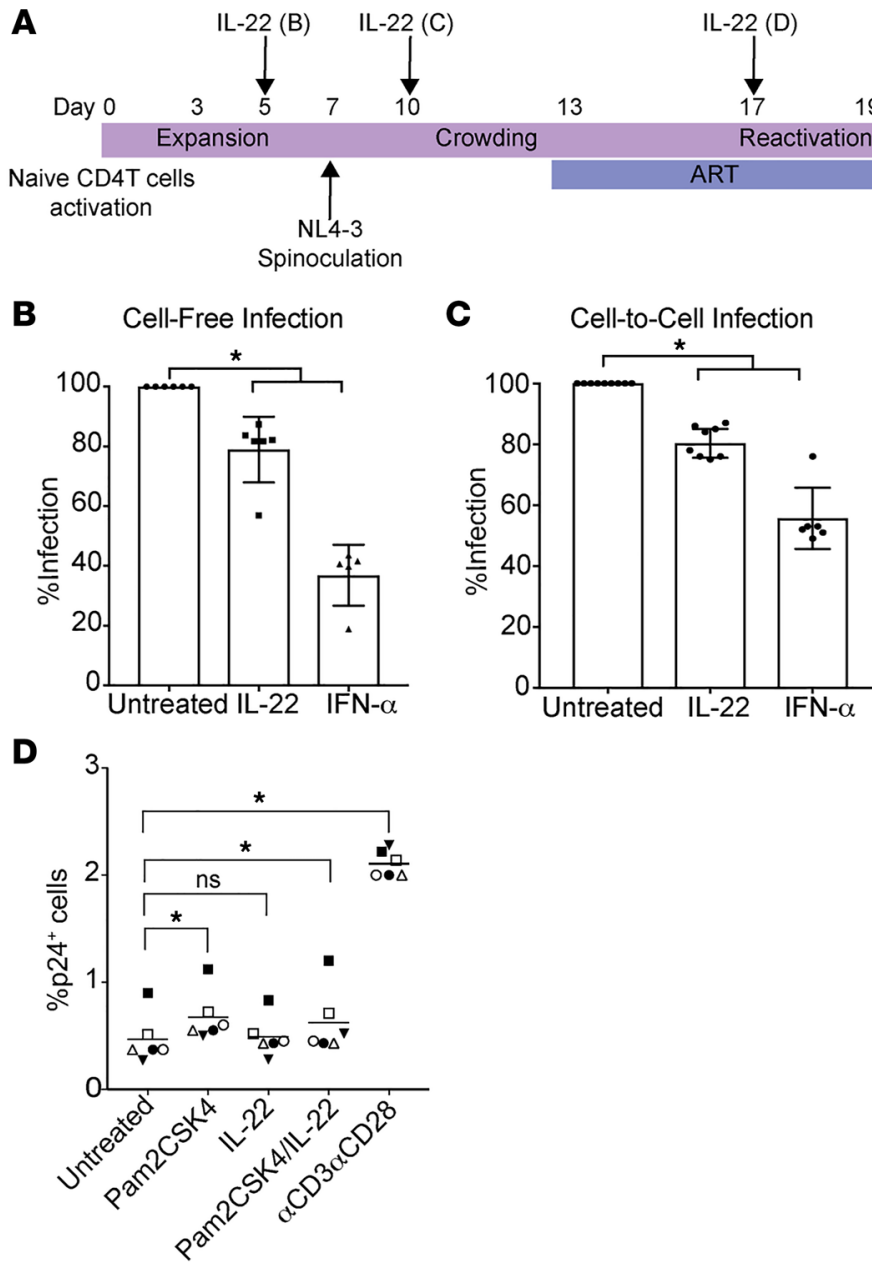

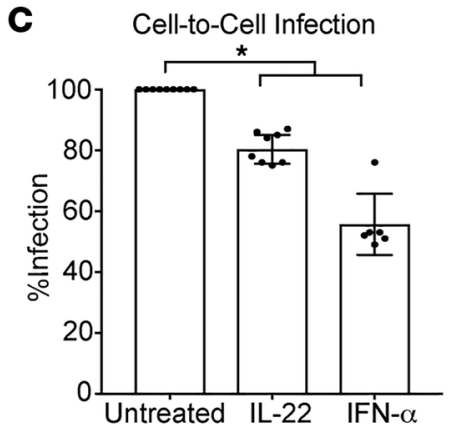

Figure 5. IL-22 promotes an antiviral state in $\mathrm{CD4}^{+} \mathbf{T}$ cells. (A) Experimental assay timeline. (B) Cells were pretreated with IL-22 or IFN- $\alpha$ for 48 hours before spinoculation and the levels of infection were measured 3 days after $(n=6)$. Data represent the mean \pm SD. (C) Cells were treated with IL-22 or IFN- $\alpha$ during the crowding phase $(n=6-10)$. Data represent the mean \pm SD. (D) Reactivation of latent HIV in the Tcm model with IL-2 alone (untreated), IL-22, $1 \mu \mathrm{M}$ Pam2CSK4, the combination of Pam2CSK4 and IL-22, or $\alpha \operatorname{CD} 3 \alpha \operatorname{CD} 28(n=6)$. ${ }^{*} P<0.05$ by 2-tailed Wilcoxon's matched-pairs signed-rank test. ns, not significant.

viral p24 Gag using the SIMOA p24 digital ELISA 4 days after activation $(52,56)$. We detected p24 antigen in supernatants from cells stimulated with the different TLR agonists on a donor-to-donor basis, but to a lower degree than that induced by PMA/ionomycin (Figure 4F). Collectively, these results indicate that dual TLR2/7 agonists have a similar cytokine expression profile as well as the ability to promote viral protein expression similarly to GS-9620.

IL-22 promotes an antiviral state in $C D 4^{+} T$ cells. In contrast with the single TLR7 agonists, the dual TLR2/7 agonists were able to induce the secretion of IL-22. IL-22 belongs to the IL-10 superfamily and its role in HIV infection is not completely understood. Because IL-22 has been shown to activate STAT1, a signal transducer for various IFNs $(57,58)$, we hypothesized that IL-22 may promote antiviral activity in $\mathrm{CD} 4^{+} \mathrm{T}$ cells. To test this hypothesis, we evaluated the effect of IL-22 in 3 different scenarios: cell-free infection, cell-to-cell infection, and latency reactivation using the Tcm model of latency (Figure 5A). First, we observed that IL-22 had a significant effect in reducing cell-free infection (mean 21\%) compared with media control but to a lesser extent than IFN- $\alpha$ (Figure 5B). Second, IL-22 was also able to reduce cell-to-cell infection (mean 19.4\%) but also to a lesser extent than IFN- $\alpha$ (Figure 5C). Finally, IL-22 did not have an effect on viral reactivation from latency, either alone or in combination with Pam2CSK4 in the Tcm model. These results suggest that IL-22 has a role in preventing de novo HIV infection. In conclusion, the use of dual TLR2/7 agonists may have benefits not only in reactivating latent HIV and enhancing immune responses but also blocking viral infection through IL-22 induction.

\section{Discussion}

Recently, the use of TLR agonists as LRAs has started to be explored in vivo. Therapeutic immunization using TLR7 stimulation has been shown to induce viral release and increased immunological responses in SIV-infected monkeys $(32,59)$. Subcutaneous injection of TLR9 in humans increased cytokine levels, activation of innate immune responses including NK cells, and RNA plasma release in ART-treated HIV infected individuals (30). 
A

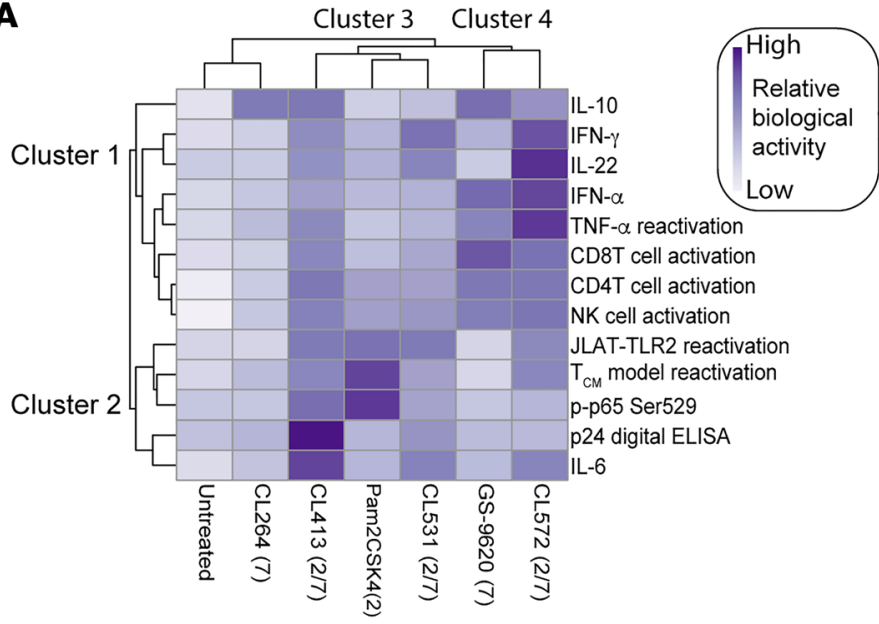

B

\begin{tabular}{llllr} 
Pam2CSK4 & CL531 & CL413 & CL572 & GS-9620 \\
\hline TLR2 & & & & TLR7
\end{tabular}

Figure 6. Heatmap visualization of the ability of each TLR agonist to have different biological activities. (A) The clustergram at the left of the heatmap reflects the relationships between the different biological activities across compounds. The clustergram at the top of the heatmap reflects the relationship between each TLR agonist across the different biological activities. Clustergrams were created using ClustVis. Dark blue cells in the heatmap reflect biological activity, whereas light blue cells indicate that the compounds do not have biological activity. (B) CL413 is the dual TLR that exhibits the best TLR2 and TLR7 agonist activity.

In this study, we have characterized in vitro the TLR2 agonist Pam2CSK4, 2 single TLR7 agonists (CL264 and GS-9620), and 4 synthetic dual TLR2/7 agonists (CL401, CL413, CL531, and CL572) (Supplemental Figure 1). To our knowledge, our study is the first one characterizing the biological function of these dual TLR2/7 agonists in human cells. Using several in vitro and ex vivo models, we have studied the ability of these agonists to reactivate latent HIV. Furthermore, we investigated the ability of these agonists to induce immune activation and the secretion of proinflammatory and antiviral cytokines. For 6 of these agonists, we performed a total of 24 assays and found biological activity in 13 of them. In order to categorize the different agonists, a heatmap was created to cluster the activity of each agonist in the different assays using ClustVis (https://biit.cs.ut.ee/clustvis/) (60). This heatmap identified 4 different main clusters (Figure 6A). Cluster 1 represents activities principally mediated by TLR7 agonists. This includes the ability of these agonists to induce the secretion of IFN- $\alpha$ or the ability of the supernatants to reactivate latent HIV in JLAT10.6, among others. Cluster 2 encompasses mainly activities mediated by TLR2 agonists including the ability to reactivate latent HIV in the Tcm model of latency, or the ability to induce phosphorylation of p65 in memory CD4 ${ }^{+} \mathrm{T}$ cells (Figure 6A). This analysis also allows us to group the different agonists. On one hand, cluster 3 includes the agonists in which activity is more TLR2-like, including Pam2CSK4, CL531, and CL413. On the other hand, cluster 4 includes agonists in which activity is more TLR7-like, including GS-9620 and CL572. These differences in activity may be due to the fact that CL431 and CL531 are closely related in structure to Pam2CSK4 and maintain most of the motifs required for binding to TLR2. In contrast, CL572 is derived from a weaker TLR2 agonist, which may allow for a better binding to TLR7. Further studies will be required to address the mechanisms by which each dual TLR2/7 agonist signals in different cell types. CL264 is a weak TLR7 agonist that lacks many of the activities measured in our study and clustered closer to the unstimulated control. Our study identified CL413 as the dual TLR2/7 agonist that preserves both the TLR2- and the TLR7-mediated biological activities, and further animal studies are required to assess whether these agonists can be used clinically (Figure 6B).

Given that TLRs are expressed in different cell compartments and can trigger diverse pathways, combining different TLR agonists can both enhance viral reactivation from latency and increase immune responses to promote anti-HIV immunity. Targeting multiple TLRs has been successfully used in other studies. It was previously shown that a nucleic acid carrier chimeric compound composed of the TLR2 agonist Pam2Cys and the TLR7 agonist CL307 had synergistic immunostimulatory properties compared with the combination of the single TLR agonists in mice (61). Here, we demonstrated that dual TLR2/7 agonists have greater ability to induce the production of soluble factors that reactivate latent HIV than the combination of a single TLR2 and TLR7 agonist (Supplemental Figure 4). Furthermore, it has been shown that the combination of different TLR agonists induces synergistic cytokine production in human and mouse DCs and macrophages when compared with single TLR agonist stimulation $(62,63)$. 
We have shown that the combination of TLR2 and TLR7 agonists in a single molecule has several advantages related to the individual agonists. First, GS-9620 is unable to reactivate latent HIV directly in CD4 ${ }^{+}$ $\mathrm{T}$ cells, while the dual TLR2/7 agonists have the ability to do so. Their relative activity is reduced relative to Pam2CSK4, probably due to the modifications present in the molecular structure of the TLR2 agonist when adding the TLR7-binding motif. However, these agonists still have activity at the low nanomolar range. Second, dual TLR2/7 agonists preserve the ability to promote TNF- $\alpha$ and to induce TNF-induced viral reactivation to levels similar to GS-9620 and superior to Pam2CSK4. Interestingly, it has been reported that GS-9620 reactivated HIV in a mechanism dependent on IFN- $\alpha$. Blocking IFN- $\alpha$ receptor 1 (IFNAR1) led to reduced $\mathrm{T}$ cell activation and HIV RNA release (28). Although our work has not demonstrated a direct role of IFN- $\alpha$ in reactivating latent $\mathrm{HIV}$ in $\mathrm{CD}^{+} \mathrm{T}$ cells, it is possible that IFN- $\alpha$ acts to induce immune activation and subsequent production of TNF- $\alpha$, as the 2 cytokines cross-regulate each other (64). Consistent with this notion, the secretion of IFN- $\alpha$ closely correlated with the ability of the supernatants to reactivate JLAT10.6 (Figure 6A). Interestingly, we did not detect TNF- $\alpha$ above the limit of detection in the supernatants from cultured PBMCs stimulated with any of the agonists after 72 hours of incubation. This is in contrast with what has been previously demonstrated for GS-9620 (28). One of the potential explanations for this finding is the timing of the analysis. The study by Tsai et al. analyzed TNF- $\alpha$ production 48 hours after stimulation (28), while we measured it at 72 hours. In fact, when TNF- $\alpha$ production was measured 24 hours after stimulation, we were able to detect induction by the different TLR agonists (Supplemental Figure 6). In line with the potential role of TNF- $\alpha$ as one of the soluble factors promoting viral reactivation, we clearly demonstrated that treatment of supernatants from PBMCs stimulated with GS-9620 or CL413 with a TNF- $\alpha$-blocking monoclonal antibody impaired viral reactivation in JLAT10.6 (Figure 2C). We also showed that this cytokine is produced by different cell types, mainly by pDCs and monocytes, after TLR stimulation (Figure 2F), indicating that TNF- $\alpha$, not IFN- $\alpha$, may be the cytokine responsible for the viral reactivation observed with TLR7 agonists. However, we do not exclude the possibility that other cytokines or soluble factors induced by TLR7 agonists may also contribute to HIV reactivation.

In this work, we have also characterized whether the ability of TLR agonists to reactivate latent HIV is influenced by the biological sex and age of the donors. This is important because several physiological differences in female and male donors have been previously described $(65,66)$. In our studies, we did not observe any major difference in either the levels of reactivation in the primary Tcm model by TLR2 agonists or the reactivation in JLAT10.6 incubated with supernatants from PBMCs stimulated with either dual TLR2/7 or TLR7 agonists. However, this study does not consider the influence of sex hormones. Recently, Das et al. demonstrated the impact that estrogens have in viral reactivation from latency (67).

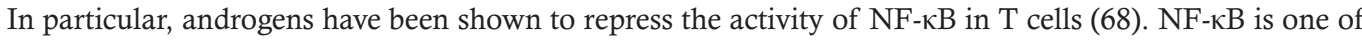
the principal HIV transcription factors and it is activated by TLR agonists $(69,70)$. It will be important to address whether estrogens impact viral reactivation mediated by TLR agonists.

We described another advantage of the dual TLR2/7 agonists relative to TLR7 agonists and it is the induction of IL-22. IL-22 is a member of the IL-10 family of cytokines, with diverse biological functions, including tissue protection, regeneration, and inflammation $(71,72)$. Furthermore, this cytokine has been implicated in antiviral responses (73-75). The role of IL-22 in HIV is still not fully characterized. Correlational studies reported gut mucosal protection during HIV infection $(76,77)$. Moreover, IL-22 could be involved in a network together with IL-10 and C-reactive protein to reduce viral replication (78). However, whether IL-22 has a direct role in protecting $\mathrm{CD} 4^{+} \mathrm{T}$ cells from HIV infection has been unknown. Here, we demonstrated a protective role in both cell-free and cell-to-cell HIV infection of $\mathrm{CD}^{+} \mathrm{T}$ cells. Future mechanistic studies are currently underway to clarify how IL-22 exerts this anti-HIV effect.

Together, these data demonstrate that dual TLR2/7 agonists can reactivate latent HIV by 2 complementary mechanisms while also stimulating diverse protective immune functions. Among the dual TLR2/ 7 agonists tested, CL413 was the one that maintained the best biological activities of both TLR2 and TLR7 agonists. Further in vivo studies in animals will need to be performed to fully test the safety and efficacy of this agonist for shock-and-kill strategies towards HIV.

\section{Methods}

Reagents. The following reagents were obtained through the AIDS Research and Reference Reagent Program, Division of AIDS, National Institute of Allergy and Infectious Diseases (NIAID): nelfinavir, raltegravir (catalog 11680) from Merck \& Company and HIV-1 ${ }_{\mathrm{NL} 4-3}$ from Malcolm Martin (catalog no. 114) (79). Raltegravir 
was also from Selleckchem. Human rIL-2 was provided by the BRB/NCI Preclinical Repository. The TLR agonists CL401, CL413, CL531, CL572, Pam2CSK4, Pam3CSK4, and CL264 were purchased from Invivogen. GS-9620, entinostat, chidamide, ICBP-112, JQ-1, PFI-1, and decitabine were purchased from Cayman Chemical Company. Recombinant cytokines IFN- $\gamma$, TNF- $\alpha$, IL-6, IL-10, IFN- $\omega$, and IP-10 were purchased from PeproTech. IFN- $\alpha$ was from Biolegend.

JLAT clones. JLAT10.6 were provided by Eric Verdin (University of California San Francisco) and JLAT-TLR2 cells were generated in our laboratory. Cell lines were maintained in RPMI 1640 media (10\% FBS, $1 \%$ penicillin/streptomycin [PS], $1 \%$ L-glutamine) at $37^{\circ} \mathrm{C}, 5 \% \mathrm{CO}_{2}$. JLAT and JLAT-TLR2 cells were plated at 50,000 cells/well in 96-well flat-bottom culture plates and incubated with single or dual TLR agonists or supernatants from PBMCs previously stimulated with TLR agonists.

Generation of latently infected cultured Tcm cells. Cultured Tcm and latently infected cultured Tcm were generated as previously described $(38,39)$.

Primary cell culture. PBMCs $\left(1 \times 10^{7}\right)$ from aviremic participants or HIV negative donors were cultured for 4 days in 12-well plates containing $3 \mathrm{ml}$ of RPMI 1640 media (10\% FBS, 1\% PS, 1\% L-glutamine) with $1 \mu \mathrm{M}$ raltegravir and $0.5 \mu \mathrm{M}$ nelfinavir, either in media control (untreated), $50 \mathrm{ng} / \mathrm{ml} \mathrm{PMA}$ and $1 \mu \mathrm{M}$ Ionomycin, or $1 \mu \mathrm{M}$ of each TLR agonist. After 48 hours of culture, $1 \times 10^{6}$ cells and $200 \mu \mathrm{l}$ of supernatant were collected for measurement of cell activation and IFN- $\alpha$ production. Seventy-two hours later, supernatant was collected to measure the accumulation of cytokine release. At 96 hours of culture, $0.5 \%$ final volume of Triton was added to $350 \mu 1$ of the supernatants, and virus was inactivated at $37^{\circ} \mathrm{C}$. Unidentified samples were sent to Quanterix for analysis of p24 using the Simoa assay $(56,80)$. Samples were analyzed in duplicate.

For experiments where isolated $\mathrm{CD}^{+} \mathrm{T}$ cells or memory $\mathrm{CD} 4^{+} \mathrm{T}$ cell were used, the EasySep Human $\mathrm{CD}^{+} \mathrm{T}$ Cell and Human Memory CD4 ${ }^{+} \mathrm{T}$ Enrichment Kit (Stem Cell) was used according to the manufacturer's instructions.

Flow cytometry. To analyze reactivation in JLAT10.6 and JLAT-TLR2, 50,000 cells were reactivated with the single and dual TLRs or filtered supernatant from PBMCs stimulated with the agonists.

To assess intracellular p24 Gag expression, $1 \times 10^{5}$ cells were fixed, permeabilized, and stained as previously described (38).

To analyze phosphorylated p65, $1 \times 10^{5} \mathrm{CD}^{+}$memory T cells were stimulated for 15 minutes at $37^{\circ} \mathrm{C}$ in the presence or absence of $1 \mu \mathrm{M}$ TLR agonists and $50 \mathrm{nM}$ PMA as positive control. After incubation, cells were washed and stained with a viability dye (Fixable Viability Dye eFluor 450, Affymetrix, eBioscience, $65-0863-18$ ) for 10 minutes at $4^{\circ} \mathrm{C}$. Cells were then fixed with $100 \mu 1$ of prewarmed $\left(37^{\circ} \mathrm{C}\right)$ Fix Buffer I (Becton Dickinson) for 10 minutes at $37^{\circ} \mathrm{C}$. Cells were washed once with $1 \mathrm{ml}$ of PBS containing $3 \%$ FBS (PBS/3\% FBS). Cells were then permeabilized while vortexing with $100 \mu 1$ of Perm Buffer III (Becton Dickinson) and incubated for 30 minutes on ice. Cells were washed once with $1 \mathrm{ml}$ of PBS/3\% FBS and stained with $2 \mu 1$ of mouse anti-phosphoserine 529 p65 conjugated to Alexa Fluor 488 (clone K10-895.12.50, catalog 558423, BD Bioscience) in $100 \mu \mathrm{l}$ of $\mathrm{PBS} / 3 \%$ FBS for 16 hours at $4^{\circ} \mathrm{C}$ for 1 hour. Finally, cells were washed once with $1 \mathrm{ml}$ of PBS/3\% FBS.

To check NK cell and T cell activation, PBMCs were removed from wells and washed with PBS/3\% FBS. Cells were stained with LIVE/DEAD Fixable Aqua Stain (Invitrogen), washed, and the following anti-human antibodies were used for staining: CD3-BV786 (clone SP34-2, catalog 563800, BD), CD4-Pacific Blue (clone RPA-T4, catalog 558116, BD), CD8-Alexa Fluor 700 (clone OKT8, catalog 56-008-42, eBioscience), CD56-BV605 (clone HCD56, catalog 318334, Biolegend), CD16-FITC (clone 3G8, catalog 555406, BD), CD14-PE (clone M5E2, catalog 555398, BD), and CD69-APCCy7 (clone FN50, catalog 310914, Biolegend). For TNF- $\alpha$ intracellular staining, $1.5 \times 10^{6}$ PBMCs from untreated, Pam2CSK4, CL413, and GS-9620 conditions received Protein Transport Inhibitor Cocktail (catalog 00-4980-03, eBioscience). The next day, cells were washed, incubated with human Fc block (catalog 564220, BD Biosciences), and then cells were stained with surface markers listed above and CD14-PB (clone HCD14, catalog 325616, Biolegend), CD123-PE (clone 6h6, catalog 306005, Biolegend), and CD303-PerCPCy5.5 (clone BDCA-2, catalog 354209, Biolegend). Finally, cells were fixed and permeabilized with Cytofix/Cytoperm buffer (catalog 554722, BD Biosciences), followed by intracellular staining with TNF- $\alpha$-APC-Cy7 (clone Mab11, catalog 502944, Biolegend).

Cells were analyzed on a BD LSR Fortessa X20 flow cytometer with FACSDIVA software (Becton Dickinson) and analyzed using FlowJo (Tree Star).

Cytokine analysis. Supernatants were collected from each well and stored at $-80^{\circ} \mathrm{C}$ until ready for 
analysis. Thirteen cytokines were measured using the LEGENDplex Human Th Cytokine Panel kit following the manufacturer's protocol (Biolegend). The following cytokines were measured in this test: IL-2, IL-4, IL-5, IL-6, IL-9, IL-10, IL-13, IL-17A, IL-17F, IL-21, IL-22, IFN- $\gamma$, and IFN- $\alpha$. IFN- $\alpha$ was quantified using a human IFN- $\alpha$ ELISA kit (R\&D Systems). For Supplemental Figure 5, single TLR and CL413 comparisons were made using the TNF- $\alpha$ ELISA Kit (catalog BMS223-4, Invitrogen).

IL-22 experiments. CD4 ${ }^{+} \mathrm{T}$ cells were treated with $10 \mathrm{ng} / \mathrm{ml} \mathrm{IL}-22$ or IFN- $\alpha$ (Peprotech) on the days indicated in the figure legend (Figure 5A).

TNF- $\alpha$-blocking experiment. Supernatants from PBMCs were collected and filtered using a $22-\mu \mathrm{m}$ syringe filter to remove cell debris and incubated with $50 \mu \mathrm{g} / \mathrm{ml}$ purified anti-TNF- $\alpha$ (clone Mab1, cat 502802, Biolegend) or isotype control IgG1 (Biolegend) for 1 hour at room temperature. Treated supernatants were cultured overnight with JLAT10.6.

PBMCs. PBMCs were isolated from buffy coats by Lymphoprep cell gradient centrifugation (STEMCELL Technologies). After being washed 3 times in PBS, the PBMCs were resuspended in RPMI 1640 medium (GE) supplemented with 10\% human FBS (VWR), 1\% L-glutamine, and 1\% PS (Gibco).

Statistics. Statistical analyses were performed using GraphPad Prism 5.0 software. Experiments were analyzed by 2-tailed Wilcoxon's matched-pairs signed-rank test when comparing stimulations, MannWhitney $U$ test (in the cases of the comparison between HIV-suppressed participants and control groups), and Spearman's correlation tests. A $P$ value less 0.05 was considered significant $\left({ }^{*} P<0.05\right.$, ${ }^{* *} P<0.01$, $\left.{ }^{* * *} P<0.001\right)$. All the data with error bars are presented as mean values $\pm \mathrm{SD}$.

Study approval. University of Utah - donors 18 years and older served as volunteer blood donors. Written informed consent was obtained from all donors. These studies are covered under protocol no. 67637 approved by the University of Utah IRB.

Gulf Coast Regional Blood Center - volunteers 17 years and older served as blood donors. White blood cell concentrate (buffy coat) prepared from a single unit of whole blood by centrifugation was purchased.

Aviremic participants - cells from aviremic participants were obtained through the Reservoir Characterization Section of the BELIEVE collaborative (IRB 021750). Secondary use of the samples was approved through George Washington University IRBs. All subjects were adults and gave informed consent.

\section{Author contributions}

$\mathrm{AB}$ and $\mathrm{ABM}$ conceived and designed the experiments. $\mathrm{ABM}$ and $\mathrm{PM}$ performed experiments. $\mathrm{ABM}$ and $\mathrm{AB}$ analyzed the data. ESS provided technical assistance. RBJ, YR, and SHH provided PBMCs from HIV-suppressed participants. CMDA performed the epigenetic screening. CLN generated the JLAT-TLR2 clone. AMS and VP provided PBMCs. AB and ABM wrote the manuscript. All the authors read and approved the manuscript.

\section{Acknowledgments}

Research reported in this publication was supported by the NIAID of the NIH under grants R01 AI124722 and R33 AI116212 (to AB) and in part by BELIEVE (NIH grant 1UM1AI26617 to RBJ) and the Reservoir Characterization Section. CMDA was supported by funds from the Brazilian Coordenação de Aperfeiçoamento de Pessoal de Nível Superior (CAPES) Foundation and the Conselho Nacional de Desenvolvimento Científico e Technológico (CNPq) as part of the Brazilian Scientific Mobility Program (BSMP). This research has been facilitated by the services and resources provided by District of Columbia, Center for AIDS research, an NIH-funded program (AI117970), which is supported by the following NIH cofunding and participating institutes and centers: NIAID, NCI, NICJD, NHLBI, NIDA, NINH, NIA, FIC, NIGGIS, NDDK, and OAR. The content is solely the responsibility of the authors and does not necessarily represent the official views of the NIH.

Address correspondence to: Alberto Bosque, School of Medicine and Health Sciences, Ross Hall, Suite 617, 2300 Eye Street NW, Washington, DC 20037, USA. Phone: 202.994.9696; Email: abosque@gwu.edu. 
CMDA's present address: Laboratório de Analises Clinicas, HU-UFJF Unidade Santa Catarina, Universidade Federal de Juiz de Fora, Juiz de Fora, Brazil.

1. Chun TW, et al. Quantification of latent tissue reservoirs and total body viral load in HIV-1 infection. Nature. 1997;387(6629):183-188.

2. Finzi D, et al. Identification of a reservoir for HIV-1 in patients on highly active antiretroviral therapy. Science. 1997;278(5341):1295-1300.

3. Rathbun RC, Lockhart SM, Stephens JR. Current HIV treatment guidelines--an overview. Curr Pharm Des. 2006;12(9):10451063.

4. Wang Z, et al. Expanded cellular clones carrying replication-competent HIV-1 persist, wax, and wane. Proc Natl Acad Sci USA. 2018;115(11):E2575-E2584.

5. Finzi $\mathrm{D}$, et al. Latent infection of $\mathrm{CD} 4^{+} \mathrm{T}$ cells provides a mechanism for lifelong persistence of HIV-1, even in patients on effective combination therapy. Nat Med. 1999;5(5):512-517.

6. Pierson T, et al. Characterization of chemokine receptor utilization of viruses in the latent reservoir for human immunodeficiency virus type 1. J Virol. 2000;74(17):7824-7833.

7. Siliciano JD, et al. Long-term follow-up studies confirm the stability of the latent reservoir for HIV-1 in resting CD4 $4^{+} \mathrm{T}$ cells. Nat Med. 2003;9(6):727-728.

8. Bosque A, Famiglietti M, Weyrich AS, Goulston C, Planelles V. Homeostatic proliferation fails to efficiently reactivate HIV-1 latently infected central memory CD4 ${ }^{+}$T cells. PLoS Pathog. 2011;7(10):e1002288.

9. Chomont N, et al. HIV reservoir size and persistence are driven by T cell survival and homeostatic proliferation. Nat Med. 2009;15(8):893-900

10. Ho YC, et al. Replication-competent noninduced proviruses in the latent reservoir increase barrier to HIV-1 cure. Cell. 2013;155(3):540-551.

11. Chun TW, Finzi D, Margolick J, Chadwick K, Schwartz D, Siliciano RF. In vivo fate of HIV-1-infected T cells: quantitative analysis of the transition to stable latency. Nat Med. 1995;1(12):1284-1290.

12. Wong JK, et al. Recovery of replication-competent HIV despite prolonged suppression of plasma viremia. Science. 1997;278(5341):1291-1295.

13. Richman DD, Margolis DM, Delaney M, Greene WC, Hazuda D, Pomerantz RJ. The challenge of finding a cure for HIV infection. Science. 2009;323(5919):1304-1307.

14. Koup RA, Hesselton RM, Safrit JT, Somasundaran M, Sullivan JL. Quantitative assessment of human immunodeficiency virus type 1 replication in human xenografts of acutely infected Hu-PBL-SCID mice. AIDS Res Hum Retroviruses. 1994;10(3):279-284.

15. Davey RT, et al. HIV-1 and T cell dynamics after interruption of highly active antiretroviral therapy (HAART) in patients with a history of sustained viral suppression. Proc Natl Acad Sci USA. 1999;96(26):15109-15114.

16. Hamer DH. Can HIV be cured? Mechanisms of HIV persistence and strategies to combat it. Curr HIV Res. 2004;2(2):99-111.

17. Jones RB, Walker BD. HIV-specific CD8 ${ }^{+} \mathrm{T}$ cells and HIV eradication. J Clin Invest. 2016;126(2):455-463.

18. Hopkins PA, Sriskandan S. Mammalian Toll-like receptors: to immunity and beyond. Clin Exp Immunol. 2005;140(3):395-407.

19. Janeway CA, Medzhitov R. Innate immune recognition. Annu Rev Immunol. 2002;20:197-216.

20. Akira S, Takeda K, Kaisho T. Toll-like receptors: critical proteins linking innate and acquired immunity. Nat Immunol. 2001;2(8):675-680.

21. Akira S, Uematsu S, Takeuchi O. Pathogen recognition and innate immunity. Cell. 2006;124(4):783-801.

22. Kawai T, Akira S. The role of pattern-recognition receptors in innate immunity: update on Toll-like receptors. Nat Immunol. 2010;11(5):373-384

23. Báfica A, Scanga CA, Schito ML, Hieny S, Sher A. Cutting edge: in vivo induction of integrated HIV-1 expression by mycobacteria is critically dependent on Toll-like receptor 2. J Immunol. 2003;171(3):1123-1127.

24. Scheller C, et al. CpG oligodeoxynucleotides activate HIV replication in latently infected human T cells. J Biol Chem. 2004;279(21):21897-21902.

25. Thibault S, Imbeault M, Tardif MR, Tremblay MJ. TLR5 stimulation is sufficient to trigger reactivation of latent HIV-1 provirus in T lymphoid cells and activate virus gene expression in central memory CD4 ${ }^{+} \mathrm{T}$ cells. Virology. 2009;389(1-2):20-25.

26. Schlaepfer E, Audigé A, Joller H, Speck RF. TLR7/8 triggering exerts opposing effects in acute versus latent HIV infection. J Immunol. 2006;176(5):2888-2895.

27. Novis CL, et al. Reactivation of latent HIV-1 in central memory CD4+ T cells through TLR-1/2 stimulation. Retrovirology. 2013;10:119.

28. Tsai A, et al. Toll-like receptor 7 agonist GS-9620 induces HIV expression and HIV-specific immunity in cells from HIV-infected individuals on suppressive antiretroviral therapy. J Virol. 2017;91(8).

29. Offersen R, et al. A novel Toll-like receptor 9 agonist, MGN1703, enhances HIV-1 transcription and NK cell-mediated inhibition of HIV-1-infected autologous CD4+ T cells. J Virol. 2016;90(9):4441-4453.

30. Vibholm L, et al. Short-course Toll-like receptor 9 agonist treatment impacts innate immunity and plasma viremia in individuals with human immunodeficiency virus infection. Clin Infect Dis. 2017;64(12):1686-1695.

31. Gane EJ, et al. The oral toll-like receptor-7 agonist GS-9620 in patients with chronic hepatitis B virus infection. $J$ Hepatol. 2015;63(2):320-328

32. Lim SY, et al. TLR7 agonists induce transient viremia and reduce the viral reservoir in SIV-infected rhesus macaques on antiretroviral therapy. Sci Transl Med. 2018;10(439).

33. Larson EC, et al. Mycobacterium tuberculosis reactivates latent HIV-1 in T cells in vitro. PLoS One. 2017;12(9):e0185162.

34. Bhat KH, Chaitanya CK, Parveen N, Varman R, Ghosh S, Mukhopadhyay S. Proline-proline-glutamic acid (PPE) protein Rv1168c of Mycobacterium tuberculosis augments transcription from HIV-1 long terminal repeat promoter. J Biol Chem. 2012;287(20):16930-16946. 
35. Buwitt-Beckmann U, et al. Toll-like receptor 6-independent signaling by diacylated lipopeptides. Eur J Immunol. 2005;35(1):282-289.

36. Agnihotri G, et al. Structure-activity relationships in toll-like receptor 2-agonists leading to simplified monoacyl lipopeptides J Med Chem. 2011;54(23):8148-8160.

37. Jordan A, Bisgrove D, Verdin E. HIV reproducibly establishes a latent infection after acute infection of T cells in vitro. EMBO J. 2003;22(8):1868-1877.

38. Martins LJ, et al. Modeling HIV-1 latency in primary t cells using a replication-competent virus. AIDS Res Hum Retroviruses. 2016;32(2):187-193.

39. Bosque A, Planelles V. Induction of HIV-1 latency and reactivation in primary memory CD4+ T cells. Blood. 2009;113(1):58-65.

40. Garcia JV, Miller AD. Serine phosphorylation-independent downregulation of cell-surface CD4 by nef. Nature. 1991;350(6318):508-511.

41. Willey RL, Maldarelli F, Martin MA, Strebel K. Human immunodeficiency virus type 1 Vpu protein induces rapid degradation of CD4. J Virol. 1992;66(12):7193-7200

42. Stoltzfus CM, Madsen JM. Role of viral splicing elements and cellular RNA binding proteins in regulation of HIV-1 alternative RNA splicing. Curr HIV Res. 2006;4(1):43-55.

43. Wang D, Baldwin AS. Activation of nuclear factor-kappaB-dependent transcription by tumor necrosis factor-alpha is mediated through phosphorylation of RelA/p65 on serine 529. J Biol Chem. 1998;273(45):29411-29416.

44. Christian F, Smith EL, Carmody RJ. The regulation of NF-кB subunits by phosphorylation. Cells. 2016;5:E12.

45. Laird GM, et al. Ex vivo analysis identifies effective HIV-1 latency-reversing drug combinations. J Clin Invest. 2015;125(5):1901-1912.

46. Bliss CI. The toxicity of poisons applied jointly. Ann Appl Biol. 1939;26(3):585-615.

47. Wightman F, et al. Entinostat is a histone deacetylase inhibitor selective for class 1 histone deacetylases and activates HIV production from latently infected primary T cells. AIDS. 2013;27(18):2853-2862.

48. Yang W, et al. Chidamide, a histone deacetylase inhibitor-based anticancer drug, effectively reactivates latent HIV-1 provirus [published online ahead of print November 8, 2017]. Microbes Infect. https://doi.org/10.1016/j.micinf.2017.10.003.

49. Zhu J, et al. Reactivation of latent HIV-1 by inhibition of BRD4. Cell Rep. 2012;2(4):807-816.

50. Lu P, et al. BET inhibitors RVX-208 and PFI-1 reactivate HIV-1 from latency. Sci Rep. 2017;7(1):16646.

51. Kauder SE, Bosque A, Lindqvist A, Planelles V, Verdin E. Epigenetic regulation of HIV-1 latency by cytosine methylation. PLoS Pathog. 2009;5(6):e1000495.

52. Bosque A, et al. Benzotriazoles reactivate latent HIV-1 through inactivation of STAT5 SUMOylation. Cell Rep. 2017;18(5):1324-1334

53. Schlaepfer E, Speck RF. TLR8 activates HIV from latently infected cells of myeloid-monocytic origin directly via the MAPK pathway and from latently infected CD4 ${ }^{+} \mathrm{T}$ cells indirectly via TNF- $\alpha$. J Immunol. 2011;186(7):4314-4324.

54. Laffont S, et al. X-Chromosome complement and estrogen receptor signaling independently contribute to the enhanced TLR7mediated IFN- $\alpha$ production of plasmacytoid dendritic cells from women. J Immunol. 2014;193(11):5444-5452.

55. Meier A, et al. Sex differences in the Toll-like receptor-mediated response of plasmacytoid dendritic cells to HIV-1. Nat Med 2009;15(8):955-959.

56. Chang L, et al. Simple diffusion-constrained immunoassay for p24 protein with the sensitivity of nucleic acid amplification for detecting acute HIV infection. $J$ Virol Methods. 2013;188(1-2):153-160.

57. Hernández PP, et al. Interferon- $\lambda$ and interleukin 22 act synergistically for the induction of interferon-stimulated genes and control of rotavirus infection. Nat Immunol. 2015;16(7):698-707.

58. O'Shea JJ, Schwartz DM, Villarino AV, Gadina M, McInnes IB, Laurence A. The JAK-STAT pathway: impact on human disease and therapeutic intervention. Annu Rev Med. 2015;66:311-328.

59. Borducchi EN, et al. Ad26/MVA therapeutic vaccination with TLR7 stimulation in SIV-infected rhesus monkeys. Nature. 2016;540(7632):284-287.

60. Metsalu T, Vilo J. ClustVis: a web tool for visualizing clustering of multivariate data using Principal Component Analysis and heatmap. Nucleic Acids Res. 2015;43(W1):W566-W570.

61. Gutjahr A, et al. Cutting Edge: A dual TLR2 and TLR7 ligand induces highly potent humoral and cell-mediated immune responses. J Immunol. 2017;198(11):4205-4209.

62. Fischetti L, Zhong Z, Pinder CL, Tregoning JS, Shattock RJ. The synergistic effects of combining TLR ligand based adjuvants on the cytokine response are dependent upon p38/JNK signalling. Cytokine. 2017;99:287-296.

63. Madan-Lala R, Pradhan P, Roy K. Combinatorial delivery of dual and triple TLR agonists via polymeric pathogen-like particles synergistically enhances innate and adaptive immune responses. Sci Rep. 2017;7(1):2530.

64. Cantaert T, Baeten D, Tak PP, van Baarsen LG. Type I IFN and TNF $\alpha$ cross-regulation in immune-mediated inflammatory disease: basic concepts and clinical relevance. Arthritis Res Ther. 2010;12(5):219.

65. Klein SL, Flanagan KL. Sex differences in immune responses. Nat Rev Immunol. 2016;16(10):626-638.

66. Addo MM, Altfeld M. Sex-based differences in HIV type 1 pathogenesis. J Infect Dis. 2014;209 Suppl 3:S86-S92.

67. Das B, et al. Estrogen receptor-1 is a key regulator of HIV-1 latency that imparts gender-specific restrictions on the latent reservoir. Proc Natl Acad Sci USA. 2018;115(33):E7795-E7804.

68. Dunn SE, et al. Peroxisome proliferator-activated receptor (PPAR)alpha expression in T cells mediates gender differences in development of T cell-mediated autoimmunity. J Exp Med. 2007;204(2):321-330

69. Nabel G, Baltimore D. An inducible transcription factor activates expression of human immunodeficiency virus in T cells Nature. 1987;326(6114):711-713.

70. Muzio M, Ni J, Feng P, Dixit VM. IRAK (Pelle) family member IRAK-2 and MyD88 as proximal mediators of IL-1 signaling Science. 1997;278(5343):1612-1615.

71. Pickert G, et al. STAT3 links IL-22 signaling in intestinal epithelial cells to mucosal wound healing. J Exp Med. 2009;206(7):1465-1472.

72. Sugimoto K, et al. IL-22 ameliorates intestinal inflammation in a mouse model of ulcerative colitis. J Clin Invest. 2008;118(2):534-544.

73. Guo H, Topham DJ. Interleukin-22 (IL-22) production by pulmonary natural killer cells and the potential role of IL-22 during 
primary influenza virus infection. J Virol. 2010;84(15):7750-7759.

74. Paget C, et al. Interleukin-22 is produced by invariant natural killer T lymphocytes during influenza A virus infection: potential role in protection against lung epithelial damages. J Biol Chem. 2012;287(12):8816-8829.

75. Dambacher J, et al. The role of interleukin-22 in hepatitis C virus infection. Cytokine. 2008;41(3):209-216.

76. Kim CJ, et al. A role for mucosal IL-22 production and Th22 cells in HIV-associated mucosal immunopathogenesis. Mucosal Immunol. 2012;5(6):670-680.

77. Fernandes SM, et al. Enteric mucosa integrity in the presence of a preserved innate interleukin 22 compartment in HIV type 1-treated individuals. J Infect Dis. 2014;210(4):630-640.

78. Missé D, et al. IL-22 participates in an innate anti-HIV-1 host-resistance network through acute-phase protein induction. $J$ Immunol. 2007;178(1):407-415.

79. Adachi A, et al. Production of acquired immunodeficiency syndrome-associated retrovirus in human and nonhuman cells transfected with an infectious molecular clone. J Virol. 1986;59(2):284-291.

80. Cabrera C, Chang L, Stone M, Busch M, Wilson DH. Rapid, Fully automated digital immunoassay for p24 protein with the sensitivity of nucleic acid amplification for detecting acute HIV infection. Clin Chem. 2015;61(11):1372-1380 